\title{
Vitamin D deficiency prevalence and risk factors among pregnant Chinese women
}

\author{
Chunfeng Yun ${ }^{1} \dagger$, Jing Chen ${ }^{1} \uparrow$, Yuna He ${ }^{2}$, Deqian Mao ${ }^{1}$, Rui Wang ${ }^{1}$, Yu Zhang ${ }^{1}$, \\ Chun Yang ${ }^{1}$, Jianhua Piao ${ }^{1}$ and Xiaoguang Yang ${ }^{1, *}$ \\ 'Department of Trace Element Nutrition, National Institute of Nutrition and Health, Chinese Center for Disease \\ Control and Prevention, Room 103, Nanwei Road No. 29, Xicheng District, Beijing 100050, People's Republic of \\ China: ${ }^{2}$ Department of Nutrition Surveillance, National Institute of Nutrition and Health, Chinese Center for Disease \\ Control and Prevention, Beijing, People's Republic of China
}

Submitted 2 April 2015: Final revision received 10 August 2015: Accepted 15 September 2015: First published online 20 November 2015

\begin{abstract}
Objective: To evaluate vitamin D deficiency prevalence and risk factors among pregnant Chinese women.

Design: A descriptive cross-sectional analysis.

Setting: China National Nutrition and Health Survey (CNNHS) 2010-2013.

Subjects: A total of 1985 healthy pregnant women participated. Possible predictors of vitamin D deficiency were evaluated via multiple logistic regression analyses. Results: The median serum 25-hydroxyvitamin D level was 15.5 (interquartile range $11.9-20 \cdot 0$, range $3 \cdot 0-51.5) \mathrm{ng} / \mathrm{ml}$, with 74.9 (95\% CI $73.0,76 \cdot 7) \%$ of participants being vitamin D deficient (25-hydroxyvitamin D $<20 \mathrm{ng} / \mathrm{ml}$ ). According to the multivariate logistic regression analyses, vitamin $\mathrm{D}$ deficiency was positively correlated with Hui ethnicity $(P=0 \cdot 016)$, lack of vitamin D supplement use $(P=0.021)$ and low ambient UVB level $(P<0.001)$. In the autumn months, vitamin D deficiency was related to Hui ethnicity $(P=0.012)$ and low ambient UVB level $(P<0 \cdot 001)$. In the winter months, vitamin $\mathrm{D}$ deficiency was correlated with younger age $(P=0 \cdot 050)$, later gestational age $(P=0.035)$, higher pre-pregnancy BMI $(P=0.019)$, low ambient UVB level $(P<0.001)$ and lack of vitamin D supplement use $(P=0 \cdot 007)$.

Conclusions: Vitamin D deficiency is prevalent among pregnant Chinese women. Residing in areas with low ambient UVB levels increases the risk of vitamin D deficiency, especially for women experiencing advanced stages of gestation, for younger pregnant women and for women of Hui ethnicity; therefore, vitamin D supplementation and sensible sun exposure should be encouraged, especially in the winter months. Further studies must determine optimal vitamin D intake and sun exposure levels for maintaining sufficient vitamin D levels in pregnant Chinese women.
\end{abstract}

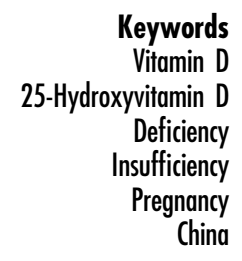

Pregnant women with low serum 25-hydroxyvitamin D $(25(\mathrm{OH}) \mathrm{D})$ levels are more likely to contract gestational diabetes, pre-eclampsia and bacterial vaginosis, and they are more likely to give birth to infants who are small for gestational age or who have a low birth weight ${ }^{(1)}$. Serum $25(\mathrm{OH}) \mathrm{D}$ levels in the fetus and the infant at birth are dependent on the maternal pool of $25(\mathrm{OH}) \mathrm{D}$ and transplacental transfer ${ }^{(2)}$. Maternal vitamin D status affects child bone-growth levels by changing periosteal bone formation patterns during childhood ${ }^{(3)}$. Additionally, lower maternal

$\dagger$ C. Yun and J. Chen contributed equally to this work and should be regarded as co-first authors. vitamin D levels may cause offspring adiposity by influencing the programming and development of muscle and fat tissue ${ }^{(4)}$. Because pregnancy serves as a critical period of fetal skeletal and nerve growth, suboptimal maternal vitamin D levels during pregnancy may have several other health consequences for the offspring ${ }^{(5)}$.

The major source of vitamin D for most human populations is sunlight exposure between approximately 09.00 and 15.00 hours (local solar time) in the spring, summer and autumn ${ }^{(6)}$. At high latitudes, solar elevation angles and ambient UVB levels are always low, and UVB exposure periods required to acquire an ideal quantity of vitamin D are sometimes impractical, especially during cooler 
seasons. Vitamin D deficiency is very common among pregnant women who live in high-latitude $\operatorname{areas}^{(7)}$. Vitamin D insufficiency is also highly prevalent in some sun-rich areas such as $\operatorname{India}^{(8)}$ and South Carolina ${ }^{(9)}$. Currently, vitamin D deficiency levels among pregnant women constitute a widespread and urgent health issue that must be remedied.

In some countries, such as the UK, pregnant women can obtain free vitamin supplements, including vitamin D supplements, from local health departments. These vitamin D supplements help raise serum 25(OH)D levels in pregnant women ${ }^{(10)}$ and may also decrease preterm birth risks $^{(11)}$. Free vitamin D supplements are not offered to pregnant women in China. Previous studies have always focused on cities or regions of China and have shown that pregnant Chinese women present a high prevalence of vitamin D deficiency or insufficiency ${ }^{(12-15)}$. Universal vitamin D screening for all pregnant Chinese women has not been reported on before.

In the present study we evaluate the prevalence of vitamin D deficiency in a nationally representative sample of pregnant women in China. We also predict the main risk factors of vitamin D deficiency among pregnant Chinese women based on age, gestational age, selfreported pre-pregnancy BMI, parity, ethnicity, ambient UVB levels in areas of residence, daily outdoor activities, vitamin D supplementation levels, smoking habits, gestational hypertension and gestational diabetes. National vitamin $\mathrm{D}$ data are instrumental to the development of health polices regarding pregnant Chinese women. The Chinese Center for Disease Control and Prevention may use these data to provide advice on vitamin D supplements for at-risk groups.

\section{Materials and methods}

\section{Study design and participants}

Data for the present analysis were taken from the China National Nutrition and Health Survey (CNNHS) 2010-2013. This survey was a nationally representative cross-sectional study that had been conducted by the Chinese Center for Disease Control and Prevention to assess the health and nutrition of Chinese civilians. It covered all thirty-one provinces, autonomous regions and municipalities directly under the central government throughout China (except Taiwan, Hong Kong and Macao). A stratified multistage probability sampling design was used in the selection of participants. The country was divided into four strata according to economic characteristics and social development. These were large cities, small to medium cities, general rural areas and poor rural areas. It involved the random selection of 150 districts (urban) or counties (rural). Serum samples were collected from pregnant women from June 2013 to March 2014. We planned to survey thirty pregnant women at random in each district or county. Briefly, approximately 4500 pregnant women were invited to participate in CNNHS 2010-2013, and 3836 participated (response rate $85 \%$ ). We excluded participants who did not have adequate serum samples for $25(\mathrm{OH}) \mathrm{D}$ measurement and those with a history of kidney disease or chronic liver disease. The final sample for the current cross-sectional analysis included $52 \%$ ( $n$ 1985) of the participants. All the participants were given a urine human chorionic gonadotrophin test. The study was conducted according to the guidelines laid down in the Declaration of Helsinki and all procedures involving human subjects were approved by the Ethics Committee of the National Institute for Nutrition and Health, Chinese Center for Disease Control and Prevention. All participants in the survey signed an informed consent form.

\section{Data collection}

Demographic data (age, ethnicity) and data on gestational age, parity, pre-pregnancy BMI, vitamin D supplement use, smoking habits and daily outdoor periods were collected based on self-reports. Questions regarding gestational diabetes symptoms were also included in the questionnaire, and all participants were asked to provide a medical report completed by a licensed doctor. Blood pressure levels were measured twice from each participant's right arm while in a seated position after 5 min of rest; the mean of the two measurements was used for analysis. Gestational hypertension was defined as a blood pressure level $>140 / 90 \mathrm{mmHg}$ at two separate times least $6 \mathrm{~h}$ apart ${ }^{(16)}$. Gestational diabetes mellitus was identified as a fasting capillary blood glucose level $>7.0 \mathrm{mmol} / \mathrm{l}^{(17)}$.

Blood samples were collected in the morning after a fasting period of $10-12 \mathrm{~h}$ on the day after the participants signed an informed consent form and were centrifuged within $0 \cdot 5-1 \mathrm{~h}$ following collection. Serum samples were then aliquoted and stored at $-80^{\circ} \mathrm{C}$ until they were analysed. Serum 25(OH)D levels were measured by a 25-Hydroxy Vitamin D EIA kit (Immunodiagnostic Systems Ltd, Boldon, UK) using a microplate reader (BioTek Synergy H1 Hybrid Reader). Inter-assay CV were 6.8 and $6.1 \%$ at 12.8 and $43.6 \mathrm{ng} / \mathrm{ml}$, respectively. In our study, participants with a serum $25(\mathrm{OH}) \mathrm{D}$ concentration of less than $12 \mathrm{ng} / \mathrm{ml}(30 \mathrm{nmol} / \mathrm{l})$ were considered to present severe levels of vitamin D deficiency; those with a concentration between 12 and $20 \mathrm{ng} / \mathrm{ml}$ were considered to present vitamin D deficiency; and those with a concentration between 20 and $30 \mathrm{ng} / \mathrm{ml}$ were considered to present vitamin D insufficiency. Serum 25(OH)D concentrations between 30 and $50 \mathrm{ng} / \mathrm{ml}$ were regarded as normal and concentrations above $50 \mathrm{ng} / \mathrm{ml}$ (particularly $>60 \mathrm{ng} / \mathrm{ml})$ may have adverse effects ${ }^{(18,19)}$.

\section{Ambient UVB measurements}

We estimated ambient UV radiation exposure levels for each participant using the Chinese administrative division 
codes of each participant's current residential address. Latitude and longitude coordinates were found for each code and were matched against UVB data available from a $1^{\circ}$ latitude $\times 1^{\circ}$ longitude grid from the NASA Goddard Space Flight Center Data Archive Center database of readings of the total ozone mapping spectrometer (TOMS) mounted on the Nimbus-7 satellite. This database was used to estimate the average erythemally weighted UVB dose $\left(\mathrm{J} / \mathrm{m}^{2}\right)$ reaching the Earth at each location from June 2013 to March 2014. The HDFtool of Matlab 7.11.0 (R2010b) was utilized to read the database. Ambient UVB levels for the entire sample were then classified into tertiles.

\section{Statistical analysis}

The participants were divided into sub-classes according to a number of hypothesized predictors for vitamin D status: region stratum, ambient UVB level of living area, season (spring, defined as March to May; summer, defined as June to August; autumn, defined as September to November; winter, defined as December to February), ethnicity, age, gestational age, parity, pre-pregnancy BMI, vitamin D supplement use, daily time spent outdoors, smoking habits, gestational hypertension and gestational diabetes. The Kolmogorov-Smirnov test was used for the normality analysis of each sub-class; most sub-classes did not follow a normal distribution. Therefore, we conducted a Kruskal-Wallis test followed by a Mann-Whitney $U$ test to examine relationships between vitamin D levels and the hypothesized predictors.

A multinomial logistic regression analysis was conducted to investigate the relationship between vitamin D deficiency levels and a number of possible predictors (i.e. age, gestational age, pre-pregnancy BMI (continuous, untransformed), parity, ethnicity, ambient UVB level of living area, daily outdoor activity periods, vitamin D supplement use, smoking habits, gestational hypertension and gestational diabetes). For the autumn and winter months, we performed an additional multinomial logistic regression to investigate the relationship between vitamin D deficiency and a number of possible predictors (i.e. age, gestational age, pre-pregnancy BMI (continuous, untransformed), parity, ethnicity, ambient UVB level of living area, daily outdoor activity periods and vitamin D supplement use). The statistical software package SAS version 9.2 was used for data analysis purposes. The primary analysis is descriptive and includes 95\% CI. The significance level was set at $P<0.05$ using twosided tests.

\section{Results}

As a part of the CNNHS in 2010-2013, serum 25(OH)D levels of 1985 pregnant women were analysed. The median age of the participants was 26.10 (interquartile range $23 \cdot 50-29 \cdot 20$, range $16 \cdot 90-43 \cdot 10$ ) years and the median gestational age of the participants was 22.00 (interquartile range $14 \cdot 00-31 \cdot 00$, range $2 \cdot 00-42 \cdot 00$ ) weeks. The median pre-pregnancy BMI level was 20.53 (interquartile range $19 \cdot 05-22.50$, range $14.02-34.45) \mathrm{kg} / \mathrm{m}^{2}$, with $16.58 \%$ of the participants being underweight $\left(\mathrm{BMI}<18.5 \mathrm{~kg} / \mathrm{m}^{2}\right), \quad 12.83 \%$ being overweight $(\mathrm{BMI}=$ $24-28 \mathrm{~kg} / \mathrm{m}^{2}$ ) and with $1.95 \%$ being obese (BMI $>28 \mathrm{~kg} /$ $\mathrm{m}^{2}$ ). Only $0.93 \%$ of the participants were classified as presenting gestational diabetes mellitus, $0.74 \%$ exhibited gestational hypertension and $73.04 \%$ of participants were nulliparous. In total, $10 \cdot 04 \%$ of the participants reported using vitamin D supplements during pregnancy. Only $0.3 \%$ of the women reported smoking while pregnant; $1.9 \%$ reported smoking prior to becoming pregnant but quitting while pregnant. The population was mainly of Han ethnicity (90.3\%). Baseline characteristics and $25(\mathrm{OH}) \mathrm{D}$ values are presented in Table 1.

The median serum 25(OH)D level was 15.5 (interquartile range $11 \cdot 9-20 \cdot 0$, range $3 \cdot 0-51 \cdot 5) \mathrm{ng} / \mathrm{ml}$. The prevalence of vitamin D deficiency $(25(\mathrm{OH}) \mathrm{D}<20 \mathrm{ng} / \mathrm{ml})$ was estimated at $74.9(95 \% \mathrm{CI} 73.0,76.7) \%$. Of these women with vitamin D deficiency, 25.5 (95\% CI $23 \cdot 5$, 27.4) \% presented severe vitamin D deficiency levels (25(OH)D $<12 \mathrm{ng} / \mathrm{ml}$ ), while $21 \cdot 1$ (95\% CI 20.9, 22.0) \% showed signs of vitamin D insufficiency $(20 \mathrm{ng} / \mathrm{ml} \leq$ $25(\mathrm{OH}) \mathrm{D}<30 \mathrm{ng} / \mathrm{ml})$.

In the logistic regression analysis for all the participants (Table 2), the following factors were associated with vitamin D deficiency (OR; 95\% CI): Hui ethnicity (1.84; $1 \cdot 12,3 \cdot 02 ; P=0 \cdot 016$, relative to Han ethnicity), ambient UVB level of living area (low: 2.52; 1.89, 3.35; $P<0.001$, medium: $1.70 ; 1.28,2 \cdot 24 ; P<0.001$, relative to high) and vitamin D supplement use (no: $1 \cdot 56 ; 1 \cdot 07,2 \cdot 28 ; P=0 \cdot 021$, relative to yes). According to a logistic regression analysis of participants whose serum was collected in the autumn months (Table 3), vitamin D deficiency was associated with Hui ethnicity $(2 \cdot 35 ; 1 \cdot 20,4.57 ; P=0.012$, relative to Han ethnicity) and ambient UVB level of living area (low: $2 \cdot 71$; 1.85, 3.97; $P<0.001$, medium: $2 \cdot 22 ; 1.49$, 3.31; $P<0.001$, relative to high). According to a logistic regression analysis of participants whose serum was collected during the winter months (Table 3), vitamin D deficiency was associated with age $(0 \cdot 96 ; 0.92,1 \cdot 00 ; P=0.050$, per year increase in age), gestational age $(1 \cdot 02 ; 1 \cdot 00,1 \cdot 04 ; P=0.035$, per unit increase in gestational age), pre-pregnancy BMI (1.09; $1 \cdot 01$, $1 \cdot 16 ; P=0.019$, per unit increase in BMI), ambient UVB level of living area (low: 5.54; 3.17, 9.68; $P<0 \cdot 001$, relative to high) and vitamin D supplement use (no: $1 \cdot 97 ; 1 \cdot 20,3 \cdot 24$; $P=0 \cdot 007$, relative to yes).

\section{Discussion}

More than half of the pregnant Chinese women participating in the present study were vitamin $\mathrm{D}$ deficient 
Table 1 Characteristics of the study participants: healthy pregnant Chinese women ( $n$ 1985), China National Nutrition and Health Survey 2010-2013

\begin{tabular}{|c|c|c|c|c|c|c|c|c|}
\hline \multirow[b]{2}{*}{ Variable/group } & \multirow[b]{2}{*}{$n$} & \multicolumn{2}{|c|}{$25(\mathrm{OH}) \mathrm{D}(\mathrm{ng} / \mathrm{ml})$} & \multirow[b]{2}{*}{$P$ value } & \multicolumn{4}{|c|}{ Percentage with 25(OH)D (ng/ml) } \\
\hline & & Mean & $95 \% \mathrm{Cl}$ & & $<12$ & $12-20$ & $20-30$ & $>30$ \\
\hline Total & 1985 & $16 \cdot 6$ & $16 \cdot 3,16 \cdot 9$ & & $25 \cdot 5$ & $49 \cdot 4$ & $21 \cdot 1$ & $4 \cdot 0$ \\
\hline Age (years) & 1985 & & & 0.001 & & & & \\
\hline$<20$ & 56 & $13 \cdot 9^{a}$ & $12 \cdot 2,15 \cdot 6$ & & $46 \cdot 4$ & $39 \cdot 3$ & $12 \cdot 5$ & 1.8 \\
\hline $20-25$ & 724 & $16 \cdot 2^{\mathrm{b}}$ & $15 \cdot 8,16 \cdot 7$ & & $27 \cdot 5$ & $50 \cdot 4$ & 18.5 & 3.6 \\
\hline $25-30$ & 787 & $16 \cdot 7^{\mathrm{b}}$ & $16 \cdot 3,17 \cdot 2$ & & 23.4 & $50 \cdot 3$ & 22.9 & 3.4 \\
\hline $30-35$ & 309 & $17 \cdot 5^{\mathrm{b}}$ & $16 \cdot 7,18 \cdot 3$ & & 23.3 & $45 \cdot 6$ & 24.6 & 6.5 \\
\hline$\geq 35$ & 109 & $16 \cdot 8^{\mathrm{b}}$ & $15 \cdot 5,18 \cdot 1$ & & $22 \cdot 9$ & $52 \cdot 3$ & $19 \cdot 3$ & 5.5 \\
\hline Gestational age & 1985 & & & 0.024 & & & & \\
\hline First trimester & 440 & $17 \cdot 0^{\mathrm{a}}$ & $16 \cdot 4,17 \cdot 6$ & & 21.6 & $52 \cdot 3$ & 21.8 & 4.3 \\
\hline Second trimester & 837 & $16 \cdot 7^{a}$ & $16 \cdot 3,17 \cdot 2$ & & 25.9 & 47.8 & $22 \cdot 3$ & 3.9 \\
\hline Third trimester & 708 & $16 \cdot 2^{b}$ & $15 \cdot 7,16 \cdot 7$ & & $27 \cdot 4$ & 49.6 & $19 \cdot 1$ & 4.0 \\
\hline Parity & 1977 & & & $<0.001$ & & & & \\
\hline 0 & 1444 & $16 \cdot 2^{A}$ & $15 \cdot 9,16.5$ & & $27 \cdot 4$ & 49.5 & 19.9 & 3.3 \\
\hline 1 & 493 & $17 \cdot 8^{\mathrm{B}}$ & $17 \cdot 2,18 \cdot 4$ & & $19 \cdot 3$ & $49 \cdot 7$ & 24.7 & $6 \cdot 3$ \\
\hline$>2$ & 40 & $15 \cdot 7^{\mathrm{A}}$ & $13.5,17.9$ & & 32.5 & 42.5 & $20 \cdot 0$ & 5.0 \\
\hline Pre-pregnancy BMI (kg/m²) & 1840 & & & 0.831 & & & & \\
\hline$<18.5$ & 302 & $16 \cdot 8$ & $16 \cdot 1,17 \cdot 6$ & & $25 \cdot 8$ & $47 \cdot 0$ & 22.5 & 4.6 \\
\hline $18 \cdot 5-24.0$ & 1288 & $16 \cdot 6$ & $16 \cdot 3,17 \cdot 0$ & & 24.7 & $50 \cdot 2$ & $21 \cdot 2$ & 4.0 \\
\hline $24.0-28.0$ & 215 & $16 \cdot 2$ & $15 \cdot 4,17 \cdot 1$ & & $26 \cdot 0$ & 54.0 & $15 \cdot 8$ & $4 \cdot 2$ \\
\hline$>28.0$ & 35 & $15 \cdot 9$ & $13 \cdot 9,18 \cdot 0$ & & 22.9 & 51.4 & $22 \cdot 9$ & 2.9 \\
\hline Region strata & 1985 & & & $<0.001$ & & & & \\
\hline Large cities & 467 & $15 \cdot 6^{\mathrm{A}}$ & $15 \cdot 1,16 \cdot 2$ & & $30 \cdot 8$ & 48.2 & $18 \cdot 8$ & $2 \cdot 1$ \\
\hline Small to medium cities & 557 & $17 \cdot 3^{B}$ & $16 \cdot 8,17.9$ & & $22 \cdot 1$ & 48.5 & 23.7 & $5 \cdot 7$ \\
\hline General rural areas & 540 & $18 \cdot 5^{\mathrm{C}}$ & $18 \cdot 0,19 \cdot 1$ & & $15 \cdot 2$ & $50 \cdot 6$ & 27.8 & 6.5 \\
\hline Poor rural areas & 421 & $14 \cdot 2^{\mathrm{D}}$ & $13 \cdot 6,14 \cdot 6$ & & 37.3 & $50 \cdot 6$ & 11.4 & 0.7 \\
\hline Vitamin D supplementation & 1972 & & & 0.134 & & & & \\
\hline No & 1792 & $16 \cdot 5$ & $16 \cdot 2,16 \cdot 8$ & & $25 \cdot 9$ & 49.4 & $20 \cdot 8$ & 3.9 \\
\hline Yes & 180 & 17.4 & $16 \cdot 0,18.5$ & & 19.4 & $51 \cdot 7$ & $23 \cdot 3$ & 5.6 \\
\hline Season & 1985 & & & $<0.001$ & & & & \\
\hline Spring & 55 & $10 \cdot 0^{\mathrm{A}}$ & $9 \cdot 3,10 \cdot 7$ & & $78 \cdot 2$ & $21 \cdot 8$ & 0.0 & 0.0 \\
\hline Summer & 50 & $18 \cdot 3^{\mathrm{B}}$ & $16 \cdot 6,20 \cdot 0$ & & 14.0 & $50 \cdot 0$ & 32.0 & 4.0 \\
\hline Autumn & 951 & $17.9^{\mathrm{B}}$ & $17 \cdot 4,18 \cdot 3$ & & $19 \cdot 2$ & 49.7 & 24.9 & $6 \cdot 1$ \\
\hline Winter & 929 & $15 \cdot 6^{C}$ & $15 \cdot 2,15 \cdot 9$ & & 29.4 & $50 \cdot 7$ & $17 \cdot 8$ & $2 \cdot 2$ \\
\hline Ambient UVB $\left(\mathrm{J} / \mathrm{m}^{2}\right)$ & 1985 & & & $<0.001$ & & & & \\
\hline Low & 788 & $15 \cdot 6$ & $15 \cdot 2,16 \cdot 0$ & & 32.5 & 48.4 & $15 \cdot 2$ & 3.9 \\
\hline Medium & 647 & $16 \cdot 2$ & $15 \cdot 7,16 \cdot 6$ & & $27 \cdot 0$ & $48 \cdot 8$ & 21.3 & $2 \cdot 8$ \\
\hline High & 550 & 18.5 & $18 \cdot 0,19 \cdot 1$ & & 13.6 & 51.6 & 29.1 & 5.6 \\
\hline Time outdoors $(\mathrm{h} / \mathrm{d})$ & 1984 & & & 0.208 & & & & \\
\hline$<0.5$ & 248 & $16 \cdot 2$ & $15 \cdot 4,17 \cdot 0$ & & 29.8 & 43.5 & 24.6 & $2 \cdot 0$ \\
\hline $0.5-1$ & 657 & 16.4 & $15 \cdot 9,16 \cdot 9$ & & 25.9 & $51 \cdot 1$ & $19 \cdot 3$ & 3.7 \\
\hline $1-2$ & 655 & $17 \cdot 0$ & $16 \cdot 5,17 \cdot 5$ & & 22.9 & $50 \cdot 8$ & $21 \cdot 1$ & $5 \cdot 2$ \\
\hline$>2$ & 424 & 16.5 & $15 \cdot 8,17 \cdot 1$ & & $26 \cdot 2$ & $48 \cdot 1$ & 21.7 & 4.0 \\
\hline Ethnicity & 1985 & & & $<0.001$ & & & & \\
\hline Han ethnicity & 1793 & $16 \cdot 8^{\mathrm{A}}$ & $16 \cdot 5,17 \cdot 1$ & & $24 \cdot 4$ & $49 \cdot 8$ & $21 \cdot 6$ & $4 \cdot 2$ \\
\hline Hui ethnicity & 42 & $10 \cdot 7^{\mathrm{B}}$ & $9 \cdot 2,12 \cdot 2$ & & $64 \cdot 3$ & 31.0 & 4.8 & 0.0 \\
\hline Other ethnicity & 150 & $16 \cdot 1^{\mathrm{A}}$ & $15 \cdot 1,17 \cdot 1$ & & $27 \cdot 3$ & $50 \cdot 0$ & $19 \cdot 3$ & 3.3 \\
\hline Smoking habits & 1984 & & & 0.666 & & & & \\
\hline Non-smoker without passive smoking & 906 & $16 \cdot 8$ & $16 \cdot 4,17 \cdot 3$ & & 24.4 & $49 \cdot 3$ & 21.5 & 4.7 \\
\hline Non-smoker with passive smoking & 1035 & $16 \cdot 4$ & $16 \cdot 0,16 \cdot 8$ & & $26 \cdot 2$ & 49.9 & 20.5 & 3.5 \\
\hline Former smoker & 38 & $15 \cdot 7$ & $13 \cdot 8,17 \cdot 7$ & & $31 . \overline{6}$ & 44.7 & $21 \cdot 1$ & 2.6 \\
\hline Current smoker & 5 & $15 \cdot 5$ & $9 \cdot 2,21.9$ & & $40 \cdot 0$ & $20 \cdot 0$ & $40 \cdot 0$ & 0.0 \\
\hline Gestational hypertension & 1892 & & & 0.104 & & & & \\
\hline No & 1878 & $16 \cdot 6$ & $16 \cdot 3,16 \cdot 9$ & & $25 \cdot 3$ & 49.5 & 21.0 & 4.2 \\
\hline Yes & 14 & 14.4 & $10 \cdot 3,18 \cdot 4$ & & $50 \cdot 0$ & $35 \cdot 7$ & $7 \cdot 1$ & $7 \cdot 1$ \\
\hline Gestational diabetes mellitus & 1836 & & & 0.940 & & & & \\
\hline No & 1819 & $16 \cdot 7$ & $16 \cdot 4,17 \cdot 0$ & & $25 \cdot 0$ & $49 \cdot 8$ & 21.0 & 4.3 \\
\hline Yes & 17 & $16 \cdot 5$ & $13 \cdot 2,19 \cdot 8$ & & $35 \cdot 3$ & 41.2 & $17 \cdot 6$ & 5.9 \\
\hline
\end{tabular}

25(OH)D, 25-hydroxyvitamin D.

a,b,c/A,B,C,D Mean values within a column with unlike superscript letters were significantly different by the Mann-Whitney $U$ test $\left({ }^{a, b, c}, P<0.05 ;{ }^{A}, B, C, D, P<0.01\right)$.

$(25(\mathrm{OH}) \mathrm{D}<20 \mathrm{ng} / \mathrm{ml})$, even during the summer and autumn seasons. The seasonal prevalence of vitamin D deficiency reached roughly $70 \%$ and $80 \%$ during autumn and winter, respectively. We observed seasonal variations in serum $25(\mathrm{OH}) \mathrm{D}$ concentrations among the participants. Serum $25(\mathrm{OH}) \mathrm{D}$ concentrations were higher in the summer and autumn months and lower in the winter and spring months. This seasonal variation in serum $25(\mathrm{OH}) \mathrm{D}$ 
Table 2 Multinomial logistic regression model for 25-hydroxyvitamin D level among healthy pregnant Chinese women ( $n$ 1668), China National Nutrition and Health Survey 2010-2013

\begin{tabular}{|c|c|c|c|c|}
\hline Variable/group & $n$ & OR & $95 \% \mathrm{Cl}$ & $P$ value \\
\hline Age & 1668 & 0.98 & $0.95,1.01$ & 0.128 \\
\hline Gestational age & 1668 & 1.01 & $1.00,1.02$ & 0.060 \\
\hline Pre-pregnancy BMI & 1668 & 1.04 & $0.99,1.08$ & 0.085 \\
\hline \multicolumn{5}{|l|}{ Parity } \\
\hline 0 & 1232 & 1.51 & $0.64,3.54$ & 0.342 \\
\hline 1 & 407 & 1.06 & $0.45,2.50$ & 0.888 \\
\hline$\geq 2$ & 29 & 1.00 & Ref. & - \\
\hline \multicolumn{5}{|l|}{ Ethnicity } \\
\hline Hui ethnicity & 122 & 1.84 & $1 \cdot 12,3.02$ & 0.016 \\
\hline Other ethnicity & 29 & 3.58 & $0.83,15 \cdot 51$ & 0.088 \\
\hline Han ethnicity & 1517 & 1.00 & Ref. & - \\
\hline \multicolumn{5}{|l|}{ Ambient UVB } \\
\hline Low & 636 & 2.52 & $1.89,3.35$ & 0.000 \\
\hline Medium & 564 & 1.70 & $1 \cdot 28,2 \cdot 24$ & 0.000 \\
\hline High & 468 & 1.00 & Ref. & - \\
\hline \multicolumn{5}{|l|}{ Daily outdoor activity } \\
\hline$<0.5 \mathrm{~h}$ & 206 & 0.87 & $0.58,1.31$ & 0.511 \\
\hline $0.5-1 \mathrm{~h}$ & 557 & 1.19 & $0.86,1.65$ & 0.288 \\
\hline $1-2 \mathrm{~h}$ & 566 & 0.97 & $0.70,1.33$ & 0.828 \\
\hline$>2 \mathrm{~h}$ & 339 & 1.00 & Ref. & - \\
\hline \multicolumn{5}{|l|}{ Vitamin D supplementation } \\
\hline No & 1519 & 1.56 & $1 \cdot 07,2 \cdot 28$ & 0.021 \\
\hline Yes & 149 & 1.00 & Ref. & - \\
\hline \multicolumn{5}{|l|}{ Smoking habits } \\
\hline Non-smoker with passive smoking & 850 & $1 \cdot 19$ & $0.94,1.50$ & 0.144 \\
\hline Current smoker & 5 & 0.59 & $0.09,3.88$ & 0.585 \\
\hline Former smoker & 32 & 1.26 & $0.53,3.01$ & 0.603 \\
\hline Non-smoker without passive smoking & 781 & 1.00 & Ref. & - \\
\hline \multicolumn{5}{|l|}{ Gestational hypertension } \\
\hline No & 1657 & 0.56 & $0.12,2 \cdot 71$ & 0.471 \\
\hline Yes & 11 & 1.00 & Ref. & - \\
\hline \multicolumn{5}{|l|}{ Gestational diabetes } \\
\hline No & 1653 & 1.22 & $0.37,4.02$ & 0.741 \\
\hline Yes & 15 & 1.00 & Ref. & - \\
\hline
\end{tabular}

Ref., reference category.

levels is consistent with previous observations of Korean adolescents $^{(20)}$. While vitamin D deficiency was highly prevalent, our results were similar to those of prior studies. A recent study conducted in south-western China found $83.6 \%$ of pregnant women to exhibit serum $25(\mathrm{OH}) \mathrm{D}$ levels of $<20 \mathrm{ng} / \mathrm{ml}^{(13)}$. Another study conducted in southeastern China showed that $68.6 \%$ of pregnant women presented serum $25(\mathrm{OH}) \mathrm{D}$ levels of $<20 \mathrm{ng} / \mathrm{ml}^{(12)}$. The highest value of $25(\mathrm{OH}) \mathrm{D}$ detected in our data was $51.5 \mathrm{ng} / \mathrm{ml}$, implying that no participants were at risk of vitamin D intoxication at levels of $>60 \mathrm{ng} / \mathrm{ml}^{(18)}$.

Most of our serum samples were gathered during the autumn and winter months. Only $5.3 \%$ of the serum samples were collected in the spring or summer. As not enough participants were recruited in the spring and summer, we focused more heavily on the main risk factors of vitamin D deficiency during the autumn and winter. For the autumn samples, low ambient UVB levels and Hui ethnicity were independent predictors for vitamin D deficiency among the sample of pregnant Chinese women. For samples collected during the winter, vitamin D deficiency levels were correlated with younger age, later gestational age, higher pre-pregnancy BMI levels and low ambient UVB levels. Differences between predictors of vitamin D deficiency for the autumn and winter seasons may be attributable to different ambient UVB levels occurring during these two seasons. Ambient UVB levels were higher in the autumn than in the winter.

Women living in areas of high ambient UVB levels exhibited higher vitamin D levels than women living in areas of low or medium ambient UVB levels. As skin exposure to UVB rays serves as a primary source of vitamin D for most people ${ }^{(6)}$, ambient UVB levels were an expected predictor of vitamin D deficiency in our study. Former studies have shown that vitamin D deficiency is more prevalent among individuals living in high-latitude areas where ambient UVB levels are always low ${ }^{(21-23)}$. Webb and Engelsen's study suggested that the exposure period needed to reach an ideal vitamin D level increases with increasing latitude and that individuals living at extreme latitudes are not able to produce enough vitamin D via their skin following exposure to UVB, particularly during the winter months ${ }^{(6)}$.

Our results showed that women of Hui ethnicity exhibited lower vitamin D levels than women of Han or other ethnicities $(10 \cdot 7 v \cdot 16 \cdot 8 \%)$, particularly in the autumn 
Table 3 Multinomial logistic regression model for 25-hydroxyvitamin D level in autumn and winter among healthy pregnant Chinese women (n 1731), China National Nutrition and Health Survey 2010-2013

\begin{tabular}{|c|c|c|c|c|c|c|c|c|}
\hline \multirow[b]{2}{*}{ Variable/group } & \multicolumn{4}{|c|}{ Autumn ( $n$ 848) } & \multicolumn{4}{|c|}{ Winter ( $n$ 883) } \\
\hline & $n$ & OR & $95 \% \mathrm{Cl}$ & $P$ value & $n$ & OR & $95 \% \mathrm{Cl}$ & $P$ value \\
\hline Age & 848 & 0.98 & $0.94,1.01$ & 0.183 & 883 & 0.96 & $0.92,1.00$ & 0.050 \\
\hline Gestational age & 848 & 1.01 & $0.99,1.02$ & 0.408 & 883 & 1.02 & $1.00,1.04$ & 0.035 \\
\hline \multicolumn{9}{|l|}{ Parity } \\
\hline 0 & 606 & 1.22 & $0.42,3.58$ & 0.717 & 653 & 1.42 & $0.34,5.90$ & 0.629 \\
\hline 1 & 222 & 1.02 & $0.35,2.99$ & 0.971 & 218 & 1.02 & $0.25,4.24$ & 0.978 \\
\hline$\geq 2$ & 20 & 1.00 & Ref. & - & 12 & 1.00 & Ref. & - \\
\hline \multicolumn{9}{|l|}{ Ethnicity } \\
\hline Hui ethnicity & 16 & 2.35 & $1.20,4.57$ & 0.012 & 18 & 1.18 & $0.58,2.37$ & 0.652 \\
\hline Other ethnicity & 59 & 5.49 & $0.69,43.47$ & 0.106 & 72 & 1.50 & $0.19,12.09$ & 0.704 \\
\hline Han ethnicity & 773 & 1.00 & Ref. & - & 793 & 1.00 & Ref. & - \\
\hline \multicolumn{9}{|l|}{ Ambient UVB } \\
\hline Low & 370 & 2.71 & $1.85,3.97$ & 0.000 & 271 & 5.54 & $3.17,9.68$ & 0.000 \\
\hline Medium & 267 & $2 \cdot 22$ & $1.49,3.31$ & 0.000 & 305 & 1.25 & $0.86,1.84$ & 0.245 \\
\hline High & 211 & 1.00 & Ref. & - & 307 & 1.00 & Ref. & - \\
\hline \multicolumn{9}{|l|}{ Daily outdoor activity } \\
\hline$<0.5 \mathrm{~h}$ & 92 & 0.93 & $0.53,1.61$ & 0.785 & 120 & 0.61 & $0.33,1.14$ & 0.124 \\
\hline $0.5-1 \mathrm{~h}$ & 280 & 1.21 & $0.78,1.88$ & 0.384 & 306 & 1.06 & $0.63,1.77$ & 0.825 \\
\hline $1-2 \mathrm{~h}$ & 291 & 1.03 & $0.68,1.57$ & 0.884 & 292 & 0.83 & $0.51,1.35$ & 0.448 \\
\hline$>2 \mathrm{~h}$ & 185 & 1.00 & Ref. & - & 165 & 1.00 & Ref. & - \\
\hline \multicolumn{9}{|c|}{ Vitamin D supplementation } \\
\hline No & 777 & 1.32 & $0.77,2.25$ & 0.307 & 803 & 1.97 & $1 \cdot 20,3 \cdot 24$ & 0.007 \\
\hline Yes & 71 & 1.00 & Ref. & - & 80 & 1.00 & Ref. & - \\
\hline
\end{tabular}

Ref., reference category.

months. This finding is attributable to the unique clothing style adopted by Hui women, who are accustomed to wearing a veil and long cloth that exposes only their eyes. In the autumn months, when ambient UVB levels are high, this clothing affects Hui women's vitamin D levels. However, during the winter months, this clothing, according to our data, does not appear to affect vitamin D levels, potentially because ambient UVB levels are depressed during the winter. Additionally, clothing style may not be a major predictor of vitamin D deficiency levels under low ambient UVB level conditions. Saudi Arabian researchers have also found that vitamin $\mathrm{D}$ levels can be affected by clothing styles ${ }^{(24)}$.

Pregnant women in their third trimester exhibited lower vitamin D levels than women in their first trimester of gestation $(16.2 v .17 \cdot 0 \mathrm{ng} / \mathrm{ml}, P=0.0243)$ in our study. Holmes et al. also found that women at 20 and 35 weeks of gestation showed lower vitamin D levels than women at 12 weeks of gestation ${ }^{(7)}$. An increased demand for vitamin $\mathrm{D}$ at early and late pregnancy stages may be a result of changes in $\mathrm{Ca}$ and vitamin $\mathrm{D}$ metabolism levels, and these changes may result from significant differences in vitamin D levels during different stages of pregnancy. In the winter months, vitamin D deficiency levels were negatively correlated with older gestational ages. Due to low ambient UVB levels in the winter months, increased demands for vitamin D during later pregnancy stages appear to be more serious.

According to our data, pregnant women who are younger than 20 years of age show lower 25-hydroxyvitamin D levels than older women. According to our multinomial logistic regression, age is a risk factor of vitamin D deficiency in the winter. This association between younger age and vitamin D status may be attributable to the growth demands of young girls. This result reflects those of former studies $^{(10,25)}$. However, as our study can only assess associations, more research is needed to determine whether younger age serves as the main cause of vitamin D deficiency for some pregnant woman. We at least know that pregnancy at a young age may not be beneficial to women's health.

Prior studies have shown that the use of vitamin D supplements may raise vitamin D levels among pregnant women $^{(10)}$ while decreasing preterm birth risks ${ }^{(11)}$. We thus hypothesized that vitamin D intake serves as an important predictor of vitamin D deficiency. Unfortunately, the CNNHS database does not detail information on total daily vitamin D intake via foods and supplements. Thus, we examined vitamin D supplement usage in our analysis, although this analysis does not serve as an adequate assessment of total daily vitamin D intake levels. In our study, vitamin D supplement use was found to be an independent predictor of vitamin D deficiency, especially in the winter.

For pregnant women, the estimated average vitamin D requirement and RDA published by the US Institute of Medicine in 2010 were designated as 10 and $15 \mu \mathrm{g} / \mathrm{d}$ for $25(\mathrm{OH}) \mathrm{D}$ concentrations of 16 and $20 \mathrm{ng} / \mathrm{ml}$, respectively ${ }^{(18)}$. The vitamin D Dietary Reference Intake published by the Chinese Nutrition Society in 2014 was also reported as 
$10 \mu \mathrm{g} / \mathrm{d}$ for pregnant women ${ }^{(26)}$. While vitamin D supplements helped increase $25(\mathrm{OH}) \mathrm{D}$ concentrations, only 28.9 and $5.6 \%$ of our participants who were using vitamin D supplements reached optimal serum $25(\mathrm{OH}) \mathrm{D}$ levels of 20 and $30 \mathrm{ng} / \mathrm{ml}$, respectively. Additionally, only $9 \cdot 1 \%$ of our study participants used vitamin D supplements. Vitamin D supplement use is not currently very popular in China. This high prevalence of vitamin D deficiency raises two questions: (i) whether outdoor daylight hours and/or the current Dietary Reference Intake of vitamin D are sufficient to maintain adequate vitamin D levels in pregnant Chinese women; and (ii) whether universal vitamin D supplementation for pregnant Chinese women is needed.

The present study is the first report on the vitamin D status of pregnant Chinese women based on a nationally representative sample. In our study, vitamin D deficiency was found to be highly prevalent among pregnant Chinese women. Therefore, this population should be educated on the importance of vitamin D levels and on ways of obtaining enough vitamin D. Sun exposure serves as the main source of vitamin D for most people ${ }^{(6)}$. However, two former studies showed that even knowledgeable medical university students in China have little awareness of the health effects of sun exposure ${ }^{(27,28)}$. These students' attitudes and practices generally focused on reducing sun exposure and on skin protection. In China, women have been found to be more likely to limit their sun exposure levels than men ${ }^{(27)}$. Most Chinese women limit their sun exposure and use sunscreen to prevent tanning ${ }^{(28)}$. Medical students, let alone the general Chinese population, know little about the relationship between sun exposure and vitamin D health. Pregnant Chinese women should be instructed to moderately increase their sun exposure habits and to use less sunscreen in order to maintain adequate vitamin D levels. It has been documented that products with a sun protection factor of 8 sufficiently reduce cutaneous cholecalciferol (vitamin $\mathrm{D}_{3}$ ) production by $95 \%^{(29)}$. In our study we found that vitamin D deficiency was significantly correlated with UVB levels and vitamin D supplement use. We also found that women of Hui ethnicity presented a high risk of vitamin D deficiency. We believe that the novelty of these data lies in their demonstration of high vitamin D deficiency prevalence, which requires public recognition. Additionally, our study results will prove instrumental to public education on vitamin D health.

The study presents some limitations. Several studies have shown that sun exposure is an important determinant of serum $25(\mathrm{OH}) \mathrm{D}$ levels ${ }^{(30,31)}$. However, we were unable to determine the sun exposure area and period for each participant. Instead, we recorded daily outdoor periods and ambient UVB levels to estimate sun exposure levels for each participant. We found that ambient UVB levels affect serum 25(OH)D levels. Dietary Ca intake is another crucial factor that affects vitamin D levels. Some experimental studies have shown that dietary Ca deficiency can lead to secondary vitamin D deficiency ${ }^{(32)}$. We did not estimate dietary $\mathrm{Ca}$ intake levels for each participant.

Another limitation is the high rate of missing data for our participants. We must explain why so many participants were excluded from our analysis. The current study serves as one part of the CNNHS 2010-2013. As part of the CNNHS 2010-2013, we sought to assess vitamin A, vitamin D and microelement levels through serum sample analyses. As serum sample collection is difficult and requires considerable laboratory use, time and funding, we only used $52 \%$ of the serum samples collected for vitamin D testing. The other serum samples were used for vitamin A and microelement tests. However, we ensured that the distributions of sociodemographic characteristics (such as age, gestational age, region strata, parity) were equal for utilized and non-utilized samples in the present study on vitamin D. We believe that there was no selection bias between the samples included and those excluded in our study.

The study was a descriptive cross-sectional analysis. All of the results can only inform us of associations between potential predictive factors and vitamin D deficiency levels. From our data, we cannot assess the true causes of high levels of vitamin D deficiency in China. This may be another limitation of our study. While the study was cross-sectional in nature, the findings suggest that higher UVB levels and vitamin D supplement use may prevent vitamin D deficiency.

\section{Conclusion}

Vitamin D deficiency was found to be highly prevalent among pregnant Chinese women, especially among those residing in areas of low ambient UVB levels. More than half of the participants were vitamin D deficient, even in the summer and autumn months. In the autumn months, being of Hui ethnicity and residence in an area of low ambient UVB levels served as independent risk factors of vitamin D deficiency. During the winter months, vitamin D deficiency was correlated with younger age, later gestational age, higher pre-pregnancy BMI and low ambient UVB level. Vitamin D supplementation may help pregnant women maintain higher vitamin D levels during the winter months. Further studies must identify optimal outdoor activity and vitamin D intake levels needed to maintain sufficient vitamin D levels among pregnant Chinese women.

\section{Acknowledgements}

Acknowledgements: The authors thank all participants in the study and the staff working for the CNNHS 2010-2013. Financial support: This research was supported by the Special Fund for Health-scientific Research in the Public 
Interest (grant number 20120212) from the National Health and Family Planning Commission of the People's Republic of China. Without this fund, we cannot finished this research. Conflict of interest: The authors declare that they have no competing interests, personal or financial conflicts of interest. Authorship: C. Yun measured participants' serum 25(OH)D levels, performed the statistical analysis and wrote the paper. J.C. developed statistical models, measured participants' serum 25(OH)D levels, participated in coordination and revised draft manuscripts. Y.H. was responsible for data cleaning and analysis as well as revising manuscripts. D.M. participated in coordination and revised manuscripts. R.W. collected participants' serum and revised manuscripts. Y.Z. helped collect the participants' serum sample and measured 25(OH)D levels. C. Yang measured participants' serum 25(OH)D levels and revised manuscripts. J.P. participated in project management and interpretation of results. X.Y. conceived of the study and participated in its design and coordination. All authors read and approved the final manuscript. Ethics of buman subject participation: The study was conducted according to the guidelines laid down in the Declaration of Helsinki and all procedures involving human subjects were approved by the Ethics Committee of the National Institute for Nutrition and Health, Chinese Center for Disease Control and Prevention. All participants provided informed consent.

\section{References}

1. Aghajafari F, Nagulesapillai T, Ronksley PE et al. (2013) Association between maternal serum 25-hydroxyvitamin D level and pregnancy and neonatal outcomes: systematic review and meta-analysis of observational studies. BMJ $\mathbf{3 4 6}$, f1169.

2. Godang K, Froslie KF, Henriksen T et al. (2014) Seasonal variation in maternal and umbilical cord $25(\mathrm{OH})$ vitamin $\mathrm{D}$ and their associations with neonatal adiposity. Eur J Endocrinol 170, 609-617.

3. Sayers A \& Tobias JH (2009) Estimated maternal ultraviolet B exposure levels in pregnancy influence skeletal development of the child. J Clin Endocrinol Metab 94, 765-771.

4. Crozier SR, Harvey NC, Inskip HM et al. (2012) Maternal vitamin $\mathrm{D}$ status in pregnancy is associated with adiposity in the offspring: findings from the Southampton Women's Survey. Am J Clin Nutr 96, 57-63.

5. Christesen HT, Elvander C, Lamont RF et al. (2012) The impact of vitamin D in pregnancy on extraskeletal health in children: a systematic review. Acta Obstet Gynecol Scand 91, $1368-1380$.

6. Webb AR \& Engelsen O (2006) Calculated ultraviolet exposure levels for a healthy vitamin D status. Photochem Photobiol 82, 1697-1703.

7. Holmes VA, Barnes MS, Alexander HD et al. (2009) Vitamin D deficiency and insufficiency in pregnant women: a longitudinal study. BrJ Nutr 102, 876-881.

8. Sahu M, Bhatia V, Aggarwal A et al. (2009) Vitamin D deficiency in rural girls and pregnant women despite abundant sunshine in northern India. Clin Endocrinol (Oxf) 70, 680-684.

9. Hamilton SA, McNeil R, Hollis BW et al. (2010) Profound Vitamin D deficiency in a diverse group of women during pregnancy living in a sun-rich environment at latitude 32 degrees N. Int J Endocrinol 2010, 917428.

10. Ginde AA, Sullivan AF, Mansbach JM et al. (2010) Vitamin D insufficiency in pregnant and nonpregnant women of childbearing age in the United States. Am J Obstet Gynecol 202, 436 e $431-438$.

11. De-Regil LM, Palacios C, Ansary A et al. (2012) Vitamin D supplementation for women during pregnancy. Cochrane Database Syst Rev 2, CD008873.

12. Tao M, Shao H, Gu J et al. (2012) Vitamin D status of pregnant women in Shanghai, China. J Matern Fetal Neonatal Med 25, 237-239.

13. Xiang F, Jiang J, Li H et al. (2013) High prevalence of vitamin $\mathrm{D}$ insufficiency in pregnant women working indoors and residing in Guiyang, China. J Endocrinol Invest 36, 503-507.

14. Song SJ, Si S, Liu J et al. (2013) Vitamin D status in Chinese pregnant women and their newborns in Beijing and their relationships to birth size. Public Health Nutr 16, 687-692.

15. Jiang L, Xu J, Pan S et al. (2012) High prevalence of hypovitaminosis $\mathrm{D}$ among pregnant women in southeast China. Acta Paediatr 101, e192-e194.

16. Anon. (2000) Report of the National High Blood Pressure Education Program Working Group on High Blood Pressure in Pregnancy. Am J Obstet Gynecol 183, issue 1, S1-S22.

17. World Health Organization (2006) Definition and Diagnosis of Diabetes Mellitus and Intermediate Hyperglycemia: Report of a WHO/IDF Consultation. Geneva: WHO.

18. Institute of Medicine (2011) Dietary Reference Intakes for Calcium and Vitamin D. Washington, DC: National Academy of Sciences.

19. Holick MF, Binkley NC, Bischoff-Ferrari HA et al. (2011) Evaluation, treatment, and prevention of vitamin D deficiency: an Endocrine Society clinical practice guideline. J Clin Endocrinol Metab 96, 1911-1930.

20. Lee YA, Kim HY, Hong H et al. (2014) Risk factors for low vitamin D status in Korean adolescents: the Korea National Health and Nutrition Examination Survey (KNHANES) 2008-2009. Public Health Nutr 17, 764-771.

21. Johnson DD, Wagner CL, Hulsey TC et al. (2011) Vitamin D deficiency and insufficiency is common during pregnancy. Am J Perinatol 28, 7-12.

22. Kazemi A, Sharifi F, Jafari N et al. (2009) High prevalence of vitamin $\mathrm{D}$ deficiency among pregnant women and their newborns in an Iranian population. $J$ Womens Health 18, 835-839.

23. Bodnar LM, Simhan HN, Powers RW et al. (2007) High prevalence of vitamin D insufficiency in black and white pregnant women residing in the northern United States and their neonates. $J$ Nutr 137, 447-452.

24. Al-Ghamdi MA, Lanham-New SA \& Kahn JA (2012) Differences in vitamin D status and calcium metabolism in Saudi Arabian boys and girls aged 6 to 18 years: effects of age, gender, extent of veiling and physical activity with concomitant implications for bone health. Public Health Nutr 15, 1845-1853.

25. Andersen LB, Abrahamsen B, Dalgard C et al. (2013) Parity and tanned white skin as novel predictors of vitamin D status in early pregnancy: a population-based cohort study. Clin Endocrinol (Oxf) 79, 333-341.

26. Chinese Nutrition Society (2013) Chinese Dietary Reference Intakes (DRIs). Beijing: Science Press.

27. Gao Q, Liu G \& Liu Y (2014) Knowledge, attitude and practice regarding solar ultraviolet exposure among medical university students in Northeast China.J Photochem Photobiol B 140, 14-19.

28. Zhou M, Zhuang W, Yuan Y et al. (2015) Investigation on vitamin $\mathrm{D}$ knowledge, attitude and practice of university students in Nanjing, China. Public Health Nutr (Epublication ahead of print version). 
29. Matsuoka LY, Ide L, Wortsman J et al. (1987) Sunscreens suppress cutaneous vitamin $\mathrm{D}_{3}$ synthesis. J Clin Endocrinol Metab 64, 1165-1168.

30. Maeda SS, Kunii IS, Hayashi L et al. (2007) The effect of sun exposure on 25-hydroxyvitamin D concentrations in young healthy subjects living in the city of Sao Paulo, Brazil. BrazJ Med Biol Res 40, 1653-1659.
31. Brot C, Vestergaard P, Kolthoff N et al. (2001) Vitamin D status and its adequacy in healthy Danish perimenopausal women: relationships to dietary intake, sun exposure and serum parathyroid hormone. Br J Nutr 86, Suppl. 1, S97-S103.

32. Clements MR, Johnson L \& Fraser DR (1987) A new mechanism for induced vitamin D deficiency in calcium deprivation. Nature 325, 62-65. 